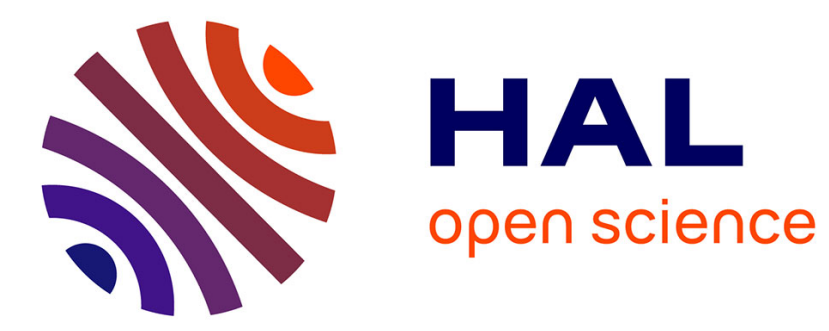

\title{
SLC37A1 Gene expression is up-regulated by epidermal growth factor in breast cancer cells
}

\author{
Domenico Iacopetta, Rosamaria Lappano, Anna Rita Cappello, Marianna \\ Madeo, Ernestina Marianna Francesco, Antonella Santoro, Rosita Curcio, \\ Loredana Capobianco, Vincenzo Pezzi, Marcello Maggiolini, et al.
}

\section{To cite this version:}

Domenico Iacopetta, Rosamaria Lappano, Anna Rita Cappello, Marianna Madeo, Ernestina Marianna Francesco, et al.. SLC37A1 Gene expression is up-regulated by epidermal growth factor in breast cancer cells. Breast Cancer Research and Treatment, 2009, 122 (3), pp.755-764. 10.1007/s10549-0090620-x . hal-00535412

\section{HAL Id: hal-00535412 \\ https://hal.science/hal-00535412}

Submitted on 11 Nov 2010

HAL is a multi-disciplinary open access archive for the deposit and dissemination of scientific research documents, whether they are published or not. The documents may come from teaching and research institutions in France or abroad, or from public or private research centers.
L'archive ouverte pluridisciplinaire HAL, est destinée au dépôt et à la diffusion de documents scientifiques de niveau recherche, publiés ou non, émanant des établissements d'enseignement et de recherche français ou étrangers, des laboratoires publics ou privés. 


\title{
SLC37A1 Gene expression is up-regulated by epidermal growth factor in breast cancer cells
}

\author{
Domenico Iacopetta $\cdot$ Rosamaria Lappano • Anna Rita Cappello • \\ Marianna Madeo • Ernestina Marianna De Francesco • Antonella Santoro • \\ Rosita Curcio • Loredana Capobianco • Vincenzo Pezzi • Marcello Maggiolini • \\ Vincenza Dolce
}

Received: 10 September 2009/Accepted: 26 October 2009/Published online: 6 November 2009

(C) Springer Science+Business Media, LLC. 2009

\begin{abstract}
Phospholipid biosynthesis exerts an important role in the proliferation of tumor cells; however, the regulation of the proteins involved in this context still remains to be fully evaluated. SLC37A1 protein belongs to a small family of sugar-phosphate/phosphate exchangers. The sequence homology with the bacterial glycerol-3-phosphate transporter (30\%) suggests that SLC37A1 might be able to catalyze an exchange of glycerol-3-phosphate against phosphate. Glycerol-3-phosphate, found in different cellular compartments, is a fundamental substrate in phospholipid biosynthesis. In the present study, we demonstrate for the first time that epidermal growth factor (EGF) transactivates SLC37A1 promoter sequence and induces SLC37A1 mRNA, and protein expression through the EGFR/MAPK/ Fos transduction pathway in ER-negative $\mathrm{SkBr} 3$ breast cancer cells. These findings were corroborated by comparable results obtained in ER-positive endometrial Ishikawa tumor cells. Interestingly, we also show that SLC37A1 protein localizes in the endoplasmic reticulum, hence supporting its possible involvement in phospholipid biosynthesis. On the basis of our data, the up-regulation of
\end{abstract}

Domenico Iacopetta and Rosamaria Lappano equally contributed to this work.

D. Iacopetta - R. Lappano - A. R. Cappello - M. Madeo ·

E. M. De Francesco - A. Santoro - R. Curcio - V. Pezzi .

M. Maggiolini $(\bowtie) \cdot$ V. Dolce $(\bowtie)$

Department of Pharmaco-Biology, University of Calabria,

87036 Rende (CS), Italy

e-mail: marcellomaggiolini@yahoo.it

V. Dolce

e-mail: vdolce@unical.it

L. Capobianco

Department of Biological and Environmental Sciences

and Technologies, University of Salento, 73100 Lecce, Italy
SLC37A1 gene expression should be included among the well-known stimulatory action exerted by EGF in breast cancer cells. In addition, further studies are required to provide evidence concerning the potential role of EGFmediated SLC37A1 induction in breast tumor cells.

Keywords Breast cancer cells · EGF · EGFR · SLC37A1

\section{Introduction}

Fatty acid synthase (FAS) is the sole protein in the human genome capable of reductive de novo synthesis of fatty acids from acetyl-CoA [1]. Fatty acids are activated by acyl-CoA synthetases (ACS) and converted to acyl-CoA [2], which is a necessary substrate for phospholipid biosynthesis. The first step of this metabolic pathway is the formation of lysophosphatidic acid (LPA) from both glycerol-3-phosphate (G3P) and acyl-CoA. The reaction is catalyzed by glycerol-3-phosphate acyltransferase (GPAT) which is thought to be a rate-limiting step for phospholipid biosynthesis [3]. In mammals, GPAT exists in at least four isoforms, two of which are localized in the mitochondria (designated GPAT1 and GPAT2) and two localized in the endoplasmic reticulum (GPAT3 and GPAT4) [4]. The protein encoded by the SLC37A1 gene, which belongs to the SLC37 family, has been indicated as a possible candidate for the transport of G3P in the endoplasmic reticulum and/or mitochondria. The SLC37A1 protein shares a $30 \%$ sequence homology with the well-studied bacterial glycerol-3-phosphate transporter (glpT) [5], suggesting that this protein is likely to be an antiporter, which may catalyze an exchange of glycerol-3-phosphate for inorganic phosphate. The second step in phospholipid synthesis is the 
formation of phosphatidic acid from LPA. The reaction is catalyzed by lysophosphatidic acid acyltransferase (LPAAT), which has been identified in eight distinct isoforms [6].

An altered metabolism is one of the most important features in cancer cells [7]. Initially, Otto Warburg observed enhanced anaerobic glycolysis in tumor cells [8, 9], thereafter alterations in lipid metabolism have also been described [10]. The elevated glucose catabolism observed in cancer cells produces an excess of pyruvate that can be converted into lactate or into acetyl-CoA, leading to the formation of citrate within the mitochondria. Citrate is then transported into the cytosol, where it is used as a substrate for fatty acid biosynthesis.

Fatty acid synthase (FAS) overexpression has been observed in a wide variety of human tumor types [11] as a consequence of an altered transcriptional regulation [12]. In addition to FAS, several other enzymes involved in lipid metabolism have recently been shown to be implicated in tumor growth and viability [13]. In mammals, two ACS isozymes, ACSL4 and ACSL5, GPAT enzymes, and the LPAAT- $\beta$ isoform are all overexpressed by a number of human cancers cells, including glioma, lung, breast, and colon carcinoma [14-17].

Among the enzymes involved in lipid biosynthesis in tumor cells, the most studied is FAS, which is regulated by different growth factor signaling, including epidermal growth factor (EGF), as well as by steroid hormoneinduced transduction pathways, including estradiol (E2) and its cognate receptor (ER) [13, 18]. Downstream of the growth factors and steroid receptors, the phosphatidylinositol-3-kinase (PI3K)/Akt, and mitogen-activated protein kinase (MAPK) signaling pathways have been suggested to mediate FAS expression through the sterol regulatory element-binding protein 1c (SREBP-1c) [13, 18]. However, the up-regulation of FAS by activated growth factor receptors requires a complex signaling network [19, 20]. Indeed, recent reports have shown that FAS expression is controlled not only by SREBP-1c but also by other transcription factors, such as the p53 family proteins and the lipogenesis-related nuclear protein, SPOT14, which is often overexpressed in breast tumor [21, 22]. As described above, the LPAAT- $\beta$ catalyzes the acetylation of LPA, which in turn generates PA, known to act as a second messenger through the Ras/Raf/Erk and the AKT/mTOR pathways in cancer cells $[16,17,23]$. Taken together, these observations suggest that several proteins, such as ACS, GAPT, LPAAT, and FAS along with SLC37A1, could be involved in the regulation of lipid synthesis in cancer cells.

In the present study, we demonstrate for the first time that EGF up-regulates SLC37A1 mRNA and protein expression in ER-negative $\mathrm{SkBr} 3$ breast cancer cells. Moreover, we provide evidence regarding SLC37A1 localization in the endoplasmic reticulum network, corroborating its potential involvement in the phospholipid biosynthesis.

\section{Materials and methods}

\section{Reagents}

Estradiol (E2), EGF, IGF-I, and H89 were purchased from Sigma-Aldrich Corp. (Milan, Italy). AG1478 (AG), PD98059 (PD) were purchased from Biomol Research Laboratories, Inc. (DBA, Milan, Italy), and GF109203X (GFX) from Calbiochem (VWR International, Milan, Italy). All compounds were solubilized in dimethylsulfoxide, except E2 which was dissolved in ethanol.

\section{Cell culture}

SkBr3 breast cancer cells, Ishikawa endometrial cancer cells, and LNCaP prostate cancer cells were maintained in RPMI 1640, DMEM, and RPMI 1640, respectively, without phenol red (except LNCaP cells, which were maintained in medium with phenol red) supplemented with $10 \%$ fetal bovine serum (FBS). Cells were switched to medium without serum the day before experiments for immunoblots, real-time PCR (RT-PCR), and microscopy evaluation.

\section{Plasmids}

In order to generate the luciferase expression vector for the SLC37A1-5' flanking region (SLC37A1-c1), a 1.7-kb fragment $(-2148$ to -463 bp respect to the translation initiation site) was amplified from genomic human DNA by nested polymerase chain reaction [24] using the following oligonucleotide primer pairs: sense $5^{\prime}$-CTCACTATCATTCCTC CATTGAACATCT- $3^{\prime}$ and antisense $5^{\prime}$-TATGGGAAGGG CCTCTGTGG-3'; nested-sense 5'-ACCTCCTAAGCCCC ACCACA- $3^{\prime}$ and nested-antisense $5^{\prime}$-GGATAATGGGAAT GCCTCGC- $3^{\prime}$. The nested-sense and nested-antisense oligonucleotide primers were synthesized with additional $5^{\prime}-K p n I$ and $5^{\prime}-X h o I$, respectively. The genomic DNA was extracted from $\mathrm{SkBr} 3$ cells by Trizol reagent as suggested by the manufacturer (Invitrogen, Milan, Italy). PCR primer pairs were selected analyzing the $5^{\prime}$-flanking region of SLC37A1 gene in chromosome 21, location 21q22.3. The nested PCR amplification was performed, as described in [24]. The amplified product, $1.7 \mathrm{~kb}$ in length, was purified (with Qiaquick from Qiagen, Milan, Italy), cloned into the XTL luciferase reporter vector, and the construct was transformed into Escherichia coli TG1 cells. The sequence of insert was verified. A computer search of transfactor databases (www.gene-regulation.com/pub/programs/alibaba2/index) with 
the $-2148 /-463$ region of the SLC37A1 gene revealed 7 AP-1 sites listed as follows: $-1999 /-1893,-1831 /-1826$, $-1693 /-1687,-1259 /-1253,-1151 /-1145,-769 /-763$, and $-747 /-741$ (Fig. 1a). The SLC37A1 was used as template to generate the constructs deleted in different AP-1 sites. The following plasmids were generated: SLC37A1-c2 (858 bp length, containing four AP-1 sites), SLCFig. 1b). The sense and antisense oligonucleotide primers were synthesized with additional 5'-KpnI and $5^{\prime}$-XhoI, respectively. The digested PCR products were cloned directionally into the KpnI/XhoI sites of XTL. The oligonucleotide primers used to generate each fragment are listed in Table 1. Furthermore, the SLC37A1-c6 was used as template to generate the plasmid SLC37A1-c8 (247 bp length, containing no AP-1 site), and the mutation of the AP-1 site was generated using a QuikChange XL sitedirected mutagenesis kit (Stratagene, Milan, Italy). Short hairpin (sh)RNA constructs for SLC37A1, EGFR, and the control plasmids were purchased from SABioscience Corporation (Frederick, MD, USA) and used, according to the manufacturer's recommendations.

\section{Transfection and luciferase assays}

$\mathrm{SkBr} 3$ and Ishikawa cells $\left(1 \times 10^{5}\right)$ were plated into 24-well dishes with $500 \mu$ l of regular growth medium per well the day before transfection. The medium was replaced with that lacking serum on the day of transfection performed using Fugene 6 reagent as recommended by the manufacturer (Roche Diagnostics, Milan, Italy) with a mixture containing $500 \mathrm{ng}$ of each SLC37A1 construct and $2 \mathrm{ng}$ of pRL-TK. After $5 \mathrm{~h}$, the serum-free medium containing the indicated treatments was renewed, and then cells were incubated for $18 \mathrm{~h}$. Luciferase activity was measured with the dual luciferase kit (Promega) according to the manufacturer's recommendations. Firefly luciferase values were normalized to the internal transfection control provided by the Renilla luciferase activity. The normalized relative light unit values obtained from cells treated with vehicle were set as 1-fold induction upon which the activity induced by treatments was calculated.

\section{Western blotting}

Cells were grown in 10-cm dishes and lysed in $500 \mu \mathrm{l}$ of $50 \mathrm{mM} \mathrm{NaCl}, 1.5 \mathrm{mM} \mathrm{MgCl} 2,1 \mathrm{mM}$ EGTA, $10 \%$ glycerol, $1 \%$ Triton $\mathrm{X}-100$, and $1 \%$ sodium dodecyl sulfate; a mixture of protease inhibitors containing $1 \mathrm{mM}$ aprotinin, $20 \mathrm{mM}$ phenylmethylsulfonyl fluoride, and $200 \mathrm{mM}$ sodium orthovanadate. Protein concentration was determined using Bradford reagent according to the manufacturer's recommendations (Sigma-Aldrich). Equal amounts of whole protein extract were resolved on a $10 \%$ sodium dodecyl sulfate-polyacrylamide gel transferred onto a nitrocellulose membrane (Amersham Biosciences, Milan, Italy), probed overnight at $4^{\circ} \mathrm{C}$ with the antibodies against SLC37A1 (XG-6192, purchased from ProSci Inc. Poway USA), ER $\alpha$ (F-10), ER $\beta$ (H-150), EGFR (1005), IGF-IR (7G11), c-fos (H-125), $\beta$-actin (C-2), phosphorylated ERK1/2 (E-4), and ERK2 (C-14; all are purchased from Santa Cruz Biotechnology, DBA, Milan, Italy), and then revealed using the ECL Western Blotting Analysis System (Amersham Biosciences, Milan, Italy).

\section{RT-PCR}

Total RNA was extracted using a Trizol commercial kit (Invitrogen, Milan, Italy) according to the manufacturer's protocol. RNA was quantified spectrophotometrically, and its quality was checked by electrophoresis through agarose gels stained with ethidium bromide. Only samples that were not degraded and showed that clear $18 \mathrm{~S}$ and $28 \mathrm{~S}$ bands under UV light were used for RT-PCR. Total cDNA was synthesized from the RNA by reverse transcription using the murine leukemia virus reverse transcriptase (Invitrogen) following the protocol provided by the manufacturer. The expression of selected genes was quantified by RT-PCR using Step One (TM) sequence detection system (Applied Biosystems, Milano, Italy), following the manufacturer's instructions. Gene-specific primers were designed using Primer Express version 2.0 software (Applied Biosystems). Assays were performed in triplicate, and the mean values were used to calculate expression levels, using the relative standard curve method. For SLC37A1 and the ribosomal protein 18S, which was used as a control gene to obtain normalized values, the primers were: 5'-TGGGCCCTACACACTCATCA-3' (SLC37A1 forward) and 5'-CGCGTTGCCTTTCAGACTTT-3' (SLC37A1 reverse); and 5'-GGCGTCCCCCAACTTCTTA-3' (18S forward) and 5'-GGGCATCACAGACCTGTTATT-3' (18S reverse).

\section{Chromatin immunoprecipitation}

Cells grown in $10-\mathrm{cm}$ plates were shifted for $24 \mathrm{~h}$ to medium lacking serum and then treated for $1 \mathrm{~h}$ with vehicle or $50 \mathrm{ng} / \mathrm{ml}$ EGF. Chromatin immunoprecipitation (ChIP) assay was performed as previously described [25]. The immuno-cleared chromatin was precipitated with antiSLC37A1 antibody or non-specific IgG (Santa Cruz Biotechnology, DBA). A $4 \mu$ l volume of each sample was used as template to amplify by PCR, two different fragments located next the SLC37A1-5' flanking region: a first fragment of 388 bp containing the two AP1 sites corresponding to $-1259 /-1253$ and $-1151 /-1145$ and a second fragment of $300 \mathrm{bp}$ containing the two AP1 sites corresponding to 
Fig. 1 SLC37A1 promoter deletion constructs. SLC37A1 promoter sequence (a) and deletion constructs (b) are shown. The AP-1 binding sites within the SLC37A1 promoter sequence are marked with black triangle. The fragments length is reported in parenthesis. The limits of fragments are indicated on the black lines
A

$\longrightarrow-2148$

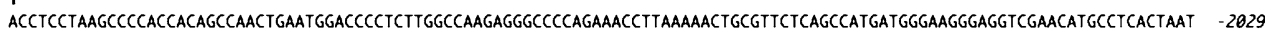

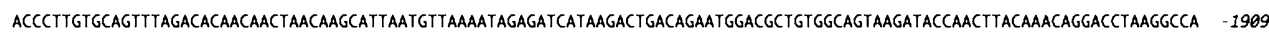

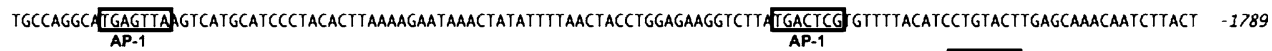

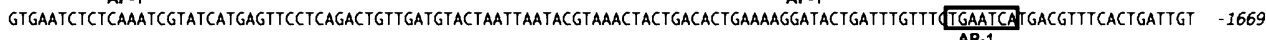

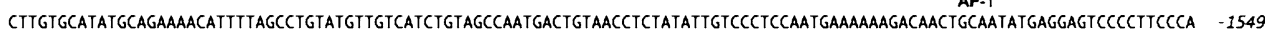

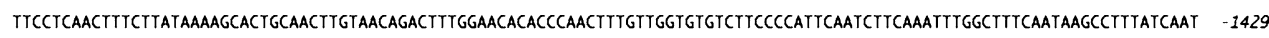

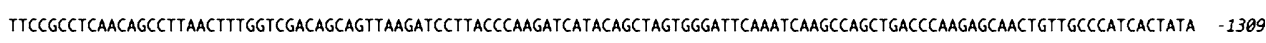

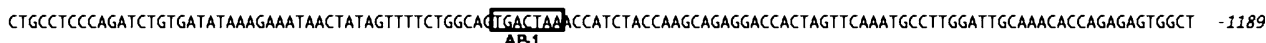

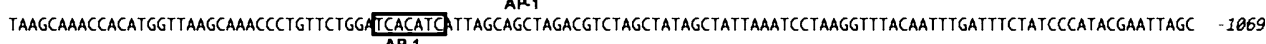

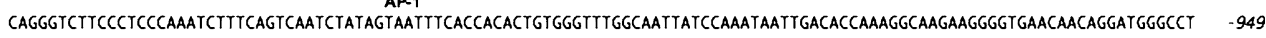

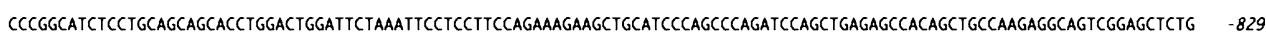

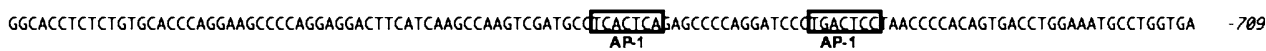
TGCCAGAAGCAGGGCTTTCCAATTCTGAaGGCCCTCAAAATGCTTGTTCAGCCCACCTTCCTCCTTTCCTGGGGGGTGGGAGTGGTGGGGAGATGAGAGGCAGAAGGATGAGGACATCAT -589

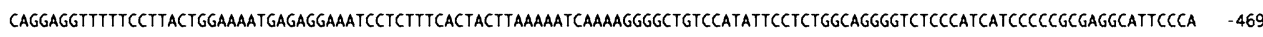
TTATCC

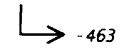

B

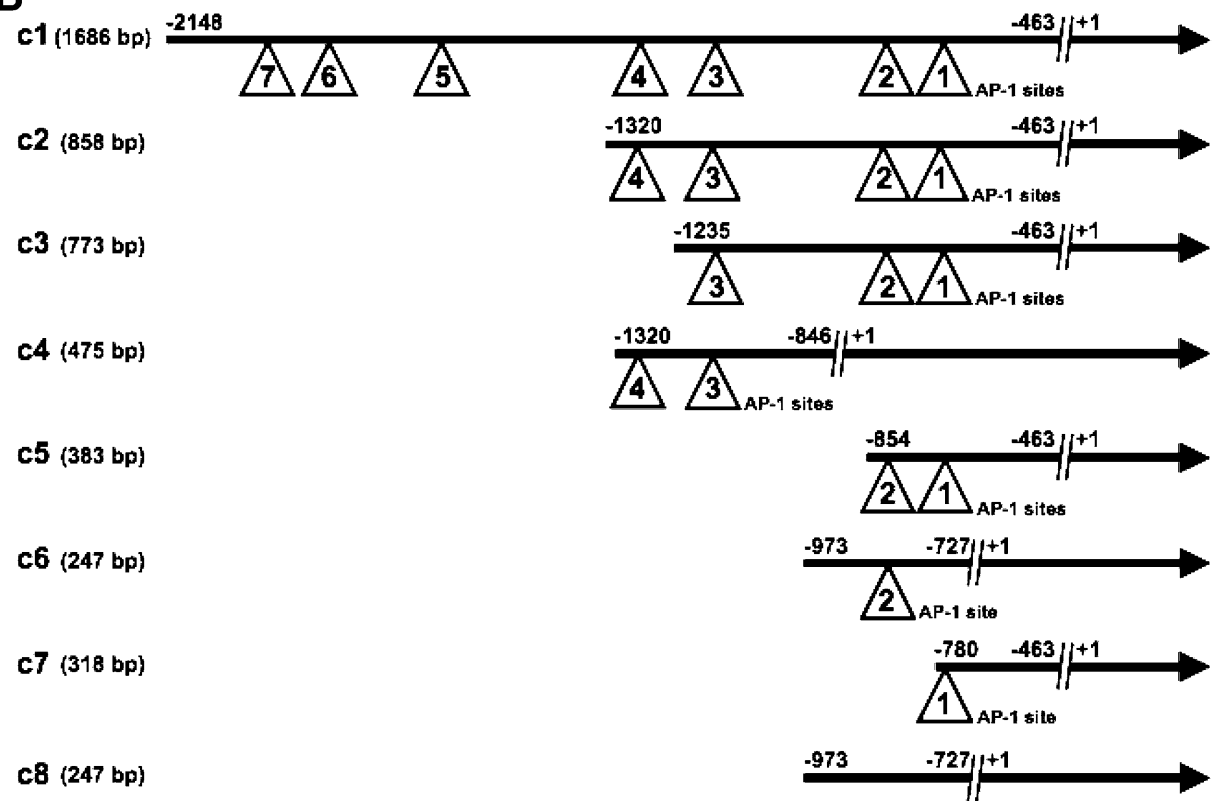

$-769 /-763$ and $-747 /-741$ of the SLC37A1 promoter sequence. The primer pairs used to amplify the first fragment were: 5'-CCGCCTCAACAGCCTTAAC-3' (forward) and $5^{\prime}$-TGACTGAAAGATTTGGGAGGG-3' (reverse), whereas the primer pairs used to amplify the second fragment were: 5'-AGAAAGAAGCTGCATCCCAG-3' (forward) and 5'-TC TTTCTGCCTCTCATCTCC-3' (reverse). The PCR conditions were $60 \mathrm{~s}$ at $95^{\circ} \mathrm{C}, 60 \mathrm{~s}$ at $60^{\circ} \mathrm{C}$, and $90 \mathrm{~s}$ at $72^{\circ} \mathrm{C}$. The amplification products obtained in 35 cycles were analyzed in a $1.2 \%$ agarose gel and visualized by ethidium bromide staining. A total of $3 \mu \mathrm{l}$ of the initial preparations of soluble chromatin were amplified to control input DNA before precipitation.
Immunofluorescence

A total of $50 \%$ confluent $\mathrm{SkBr} 3$ cells grown on coverslips were serum deprived for $24 \mathrm{~h}$. Then, cells were fixed in $4 \%$ paraformaldehyde, permeabilized with $0.2 \%$ Triton X-100, washed thrice with PBS, and non-specific sites were blocked by FBS (15\% for $30 \mathrm{~min}$ ) and incubated for over night with a chicken primary antibody against SLC37A1 (1:200; purchased from ProSci Inc. Poway, USA) and a rabbit primary antibody against calnexin (1:200; purchased from Sigma-Aldrich, Milan, Italy). After incubation with the antibodies, the slides were washed thrice with PBS and incubated with donkey anti-rabbit IgG-rodamin (1:200; 
Table 1 Synthetic oligonucleotide primers to generate the plasmids deleted in AP-1 sites

\begin{tabular}{lll}
\hline Plasmid & Oligonucleotide & Oligonucleotide primer sequence \\
\hline SLC37A1-c2 & Sense & $5^{\prime}$-CCCATCACTATACTGCCTCCCAG-3' \\
& Antisense & $5^{\prime}$-GGATAATGGGAATGCCTCGC-3' \\
SLC37A1-c3 & Sense & $5^{\prime}$-GGACCACTAGTTCAAATGCCTTGG-3' \\
& Antisense & $5^{\prime}$-GGATAATGGGAATGCCTCGC-3' \\
SLC37A1-c4 & Sense & $5^{\prime}$-CCCATCACTATACTGCCTCCCAG-3' \\
& Antisense & $5^{\prime}$-CTTGGCAGCTGTGGCTCTCA-3' \\
SLC37A1-c5 & Sense & $5^{\prime}$-AGGCAGTCGGAGCTCTGGG-3' \\
& Antisense & $5^{\prime}$-GGATAATGGGAATGCCTCGC-3' \\
SLC37A1-c6 & Sense & $5^{\prime}$-AAGGGGTGAACAACAGGATGG-3' \\
& Antisense & $5^{\prime}$-CACTGTGGGGTTAGGTAATAGG-3' \\
SLC37A1-c7 & Sense & $5^{\prime}$-AAGTCGATGCCTCTAACAGAGC-3' \\
& Antisense & $5^{\prime}$-GGATAATGGGAATGCCTCGC-3' \\
SLC37A1-c8 & Sense & $5^{\prime}$-CAAGTCGATGCCTCAAGCAGAGCCCCAGGATC-3' \\
& Antisense & $5^{\prime}$-GATCCTGGGGCTCTGCTTGAGGCATCGACTTG-3' \\
\hline
\end{tabular}

purchased from Santa Cruz Biotechnology, DBA, Milan, Italy) and rabbit anti-chicken IgG-FITC (1:200; purchased from Sigma-Aldrich, Milan, Italy). The cellular localization of SLC37A1 was evaluated by fluorescence microscope with $\times 63$ magnification. The optical sections were taken at the central plane.

Statistical analysis

Statistical analysis was done using ANOVA followed by Newman-Keuls' testing to determine differences in means. $P<0.05$ was considered as statistically significant.

\section{Results}

EGF transactivates the SLC37A1 promoter deletion constructs

Given that no data are currently available regarding the molecular mechanisms involved in the regulation of the SLC37A1 promoter sequence, we first cloned eight fragments located at the $5^{\prime}$ flanking region of the human SLC37A1 gene containing different transcription factor binding sites, including the AP-1 activating proteins (Fig. 1a, b). Thereafter, we transiently transfected, these SLC37A1 promoter deletion constructs in $\mathrm{SkBr} 3$ and Ishikawa breast and endometrial cancer cells, respectively, to evaluate the functional response of each reporter plasmid to E2 $(100 \mathrm{nM}), \mathrm{EGF}(50 \mathrm{ng} / \mathrm{ml})$, and IGF-I (50 $\mathrm{ng} / \mathrm{ml})$, agents largely involved in cancer development and progression. As shown in Fig. 2a, only EGF was able to transactivate the SLC37A1-c1 promoter construct, while the other ligands did not exhibit stimulatory activity. Next, the luciferase expression triggered by EGF was no longer observed in presence of $10 \mu \mathrm{M}$ of the EGFR and ERK inhibitors AG and $\mathrm{PD}$, respectively, while the response to EGF was not altered by $10 \mu \mathrm{M}$ of GFX and H89, inhibitors of the protein kinase $\mathrm{C}$ and protein kinase A transduction pathways, respectively (Fig. 2b). On the basis of the aforementioned results suggesting the involvement of the EGFR-ERK signaling in the transactivation of the SLC37A1-c1 by EGF, we evaluated the response of different SLC37A1 promoter deletion constructs presenting none or 1-4 AP-1 sites (Fig. 1b) potentially involved in the responsiveness to EGF. In both $\mathrm{SkBr} 3$ and Ishikawa cancer cells, $50 \mathrm{ng} / \mathrm{ml} \mathrm{EGF}$ transactivated the reporter plasmids containing at least one AP-1 site (Fig. 2ch). Therefore, the presence of only one AP-1 site is sufficient for the transactivation induced by EGF through the EGFRERK signaling as follows. In order to further characterize the functional response to EGF observed in both cell lines used, we assessed the expression of $\mathrm{ER} \alpha, \mathrm{ER} \beta, \mathrm{EGFR}$, and IGFI-R, which mainly mediate the action of E2, EGF, and IGF-I. As shown in Fig. 3, both $\mathrm{SkBr} 3$ and Ishikawa cells did not express $\mathrm{ER} \beta$, which was present in LNCaP prostate cancer cells (data not shown) used as a positive control. Ishikawa cells expressed all other receptors evaluated, while SkBr3 cells exhibited very low IGFI-R levels and lacked $\mathrm{ER} \alpha$ expression. Hence, the action of ligands tested is not dependent on the expression of the respective cognate receptors, as suggested by the response to only EGF observed in Ishikawa cells which contain all receptors except $\mathrm{ER} \beta$.

EGF up-regulates SLC37A1 expression through the EGFR-ERK transduction pathway

In order to corroborate the results obtained in transfection experiments, we evaluated SLC37A1 expression upon exposure to increasing concentrations of E2 (from 1 to 100 

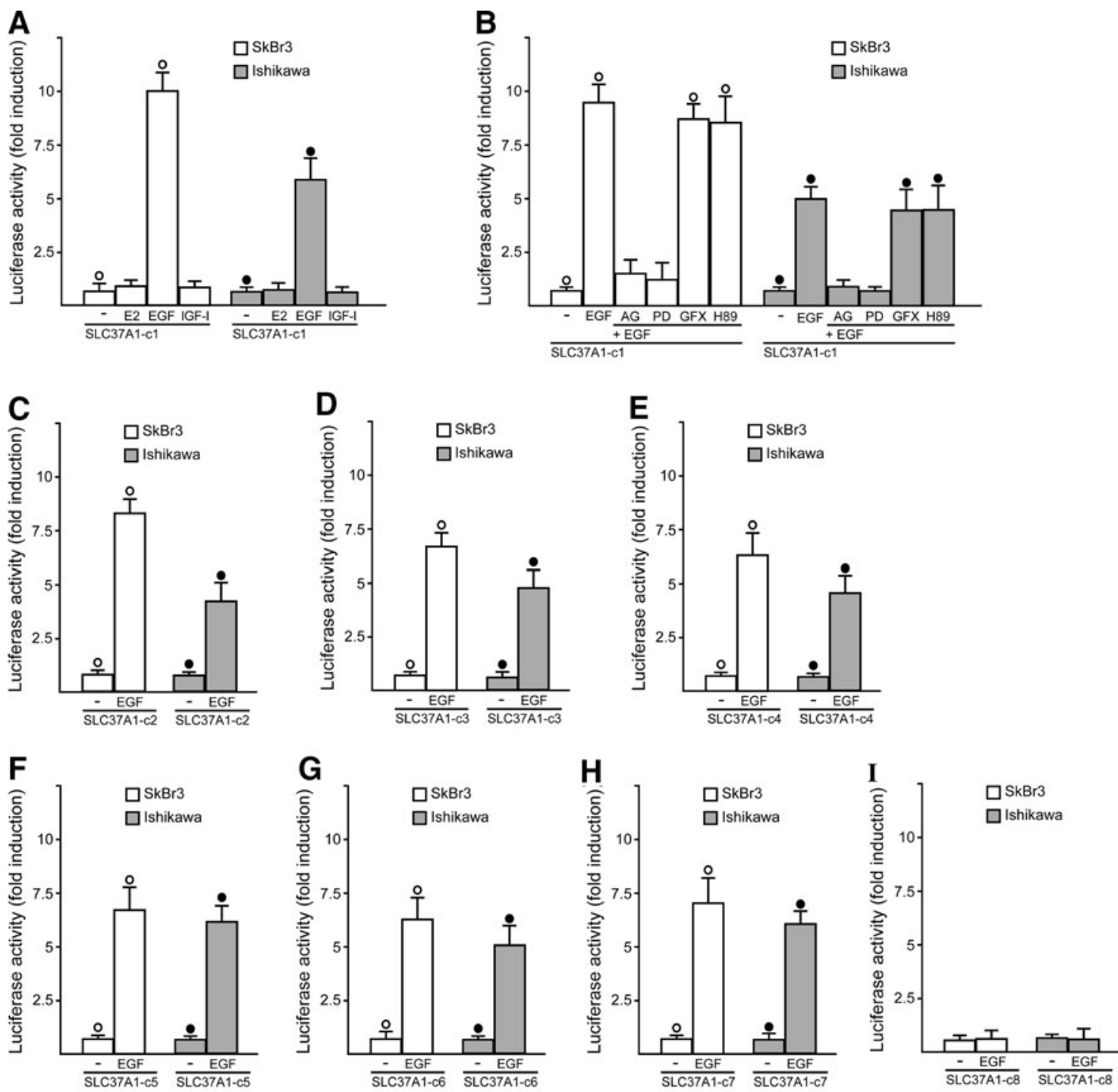

Fig. 2 EGF transactivates SLC37A1 promoter in SkBr3 and Ishikawa cells. a Cells were transfected with SLC37A1-c1 and treated with $100 \mathrm{nM} \mathrm{E2}, 50 \mathrm{ng} / \mathrm{ml} \mathrm{IGF-I,} \mathrm{or} 50 \mathrm{ng} / \mathrm{ml} \mathrm{EGF}$, as indicated. The luciferase activities were normalized to the internal transfection control, and values of cells receiving vehicle $(-)$ were set as 1 -fold induction upon which the activity induced by treatments was calculated. Each data point represents the mean \pm SD of three independent experiments performed in triplicate. b Cells were

$\mathrm{nM}$ ), EGF (from 10 to $100 \mathrm{ng} / \mathrm{ml}$ ), and IGF-I (from 10 to $100 \mathrm{ng} / \mathrm{ml}$ ). As determined by RT-PCR, only EGF upregulated in a dose-dependent manner SLC37A1 mRNA expression in $\mathrm{SkBr} 3$ cells (Fig. 4a), and in a similar extent also in Ishikawa cells (data not shown). On the basis of the aforementioned results, we treated $\mathrm{SkBr} 3$ (Fig. 4b) and Ishikawa cells (data not shown) with $100 \mathrm{nM} \mathrm{E2,} 50 \mathrm{ng} / \mathrm{ml}$ EGF, and $50 \mathrm{ng} / \mathrm{ml}$ IGF-I demonstrating that only EGF induces also SLC37A1 protein levels. As observed in transfection experiments, $10 \mu \mathrm{M}$ AG and $10 \mu \mathrm{M}$ PD prevented the SLC37A1 protein accumulation (Fig. 4c), further confirming the involvement of the EGFR/ERK

transfected with SLC37A1-c1 and treated with $50 \mathrm{ng} / \mathrm{ml}$ EGF and $10 \mu \mathrm{M}$ EGFR inhibitor tyrphostin AG 1478 (AG), $10 \mu \mathrm{M}$ MEK inhibitor PD, $10 \mu \mathrm{M}$ PKC inhibitor GFX, and $10 \mu \mathrm{M}$ PKA inhibitor H89, as indicated. $\mathbf{c}-\mathbf{h} \mathbf{S k B r} 3$ and Ishikawa cells were transfected with all SLC37A1 promoter deletion constructs cloned as indicated, and treated with $50 \mathrm{ng} / \mathrm{ml}$ EGF. Open circle, filled circle, $P<0.05$, for cells receiving vehicle (-) versus treatment

transduction pathway in the SLC37A1 induction by EGF. Next, in $\mathrm{SkBr} 3$ cells $50 \mathrm{ng} / \mathrm{ml}$ EGF stimulated ERK1/2 phosphorylation and c-fos expression which were both abrogated by $10 \mu \mathrm{M}$ AG and $10 \mu \mathrm{M}$ PD (Fig. 5a, b) or silencing EGFR expression (Fig. 5c, d), suggesting that EGFR-ERK signaling plays a crucial role in the regulation of c-fos protein levels. In order to assess whether the induction of c-fos by EGF is involved in SLC37A1 expression, we performed ChIP analysis in $\mathrm{SkBr} 3$ cells immunoprecipitating chromatin with an anti-c-fos antibody and amplifying two distinct sequences located next to the SLC37A1-5' flanking region. As shown in Fig. 6a, b, 
Fig. $3 \mathrm{ER} \alpha, \mathrm{ER} \beta, \mathrm{EGFR}$, and IGF-1R protein levels were evaluated in $\mathrm{SkBr} 3$ and Ishikawa cells. $\beta$-actin served as a loading control

A

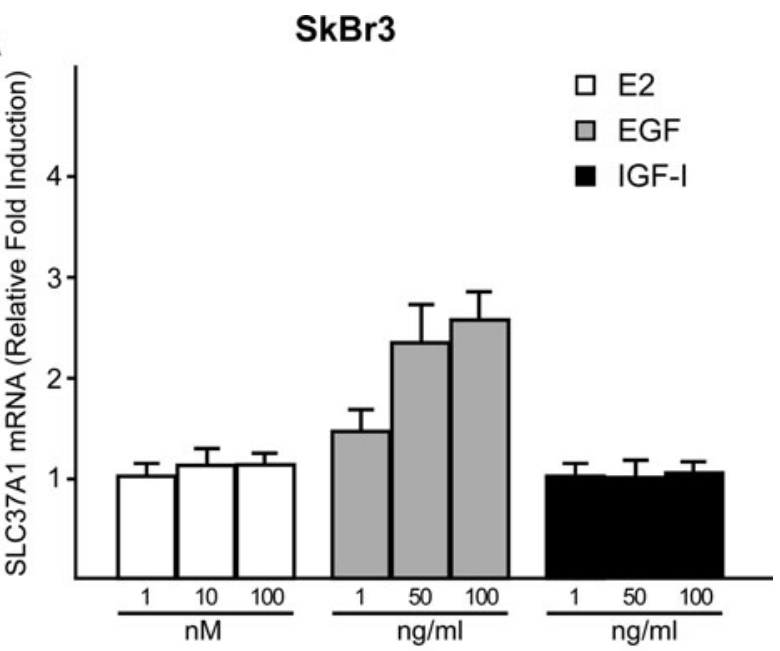

B
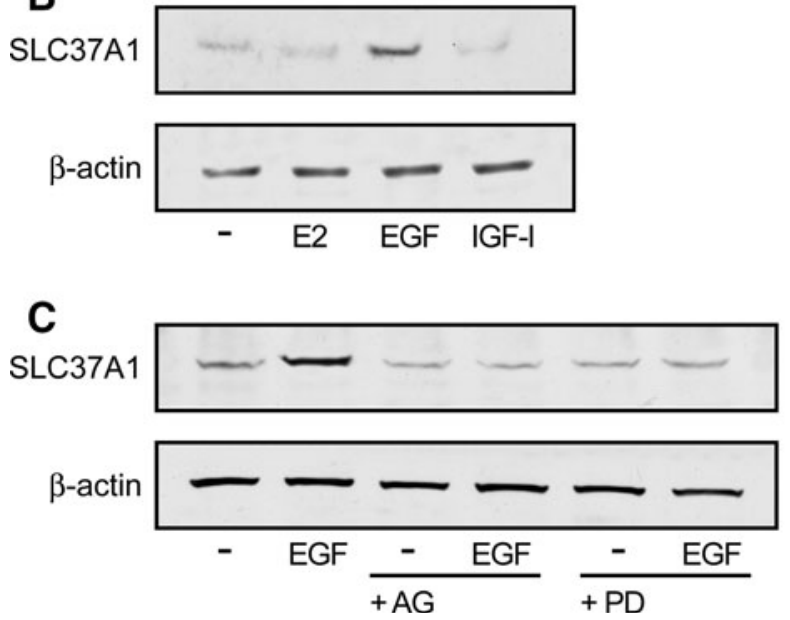

Fig. 4 EGF up-regulates SLC37A1 expression in SkBr3 cells. a The expression of SLC37A1 was evaluated by RT-PCR in $\mathrm{SkBr} 3$ cells treated for $1 \mathrm{~h}$ with increasing concentration of E2, EGF, and IGF-I, as indicated. Results obtained from experiments performed in triplicate were normalized for $18 \mathrm{~S}$ expression and shown as fold change of RNA expression compared to cells treated with vehicle. b-c Immunoblots of SLC37A1 from SkBr3 cells treated for $18 \mathrm{~h}$ with vehicle (-), $100 \mathrm{nM} \mathrm{E2,} 50 \mathrm{ng} / \mathrm{ml} \mathrm{IGF-I,} \mathrm{or} 50 \mathrm{ng} / \mathrm{ml} \mathrm{EGF} \mathrm{(b),} \mathrm{and} \mathrm{in}$ combination with $10 \mu \mathrm{M}$ EGFR inhibitor tyrphostin AG 1478 (AG) or $10 \mu \mathrm{M}$ MEK inhibitor (PD) (c), as indicated. $\beta$-actin serves as loading control treating $\mathrm{SkBr} 3$ cells with $50 \mathrm{ng} / \mathrm{ml}$ EGF c-fos was strongly recruited at the regions amplified, each containing two AP1 sites (see "Materials and methods" section). Using primer pairs amplifying a control DNA sequence which does not contain AP-1 sites, we did not detect the recruitment of c-fos (Fig. 6a, b). Taken together, these results indicate that the EGFR/ERK/c-fos transduction pathway is involved in SLC37A1 expression induced by EGF.

\section{Subcellular localization of SLC37A1}

Considering that no data are currently available regarding the intracellular localization of SLC37A1, we performed immunofluorescence studies in $\mathrm{SkBr} 3$ cells. Interestingly, the SLC37A1 staining (Fig. 7a) overlapped the pattern provided by an antibody targeting the endoplasmic reticulum network (calnexin) (Fig. 7b, c). Next, to assess the specificity of the antibody used, we knocked-down SLC37A1 protein expression by shRNA as shown in panel D of Fig. 7. The structural integrity of the cells was documented by phase contrast microscopy (data not shown). Taken together, these results suggest that the potential action(s) of SLC37A1 may be associated with the endoplasmic reticulum functions.

\section{Discussion}

The present study provides evidence regarding the regulation of SLC37A1 promoter activity in ER-negative $\mathrm{SkBr} 3$ breast cancer cells. We have demonstrated for the first time that EGF through the EGFR/ERK transduction pathway transactivates the SLC37A1-5' flanking region and up-regulates the SLC37A1 protein expression, which localizes in the endoplasmic reticulum network.

SLC37A1 belongs to the small human sugar-phosphate/ phosphate transporter family, an almost unexplored group of transmembrane sugar-phosphate/phosphate transporters consisting of four members. To date, only one of these four genes/proteins has been well-characterized: SLC37A4 protein also recognized as the glucose-6-phosphate transporter $[26,27]$. While data regarding SLC37A2 are available for mice [28], for SLC37A3 only a putative gene/protein has been identified by in silico analysis [29]. So far, data on SLC37A1 gene expression are available for man [30]. Due to its high sequence homology with the well-characterized bacterial glpT, it is very likely that the SLC37A1 protein could be a glycerol-3-phosphate/phosphate exchanger. Glycerol-3-phosphate is an essential substrate involved in several biochemical pathways, including the glycerol phosphate shuttle and phospholipid biosynthesis, which are present in distinct cellular compartments such as 
Fig. 5 EGF induces ERK1/2 phosphorylation and c-fos expression in $\mathrm{SkBr} 3$ cells. The ERK1/2 phosphorylation and cfos expression induced by $50 \mathrm{ng} / \mathrm{ml}$ EGF is abrogated in presence of $10 \mu \mathrm{M}$ EGFR inhibitor tyrphostin $\mathrm{AG}$ and $10 \mu \mathrm{M}$ MEK inhibitor PD (a-b) or silencing EGFR expression (c-d). ERK 2 and $\beta$-actin serve as loading control, respectively, e efficacy of EGFR silencing by specific shRNA

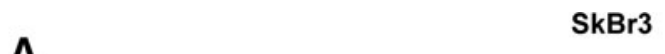

A

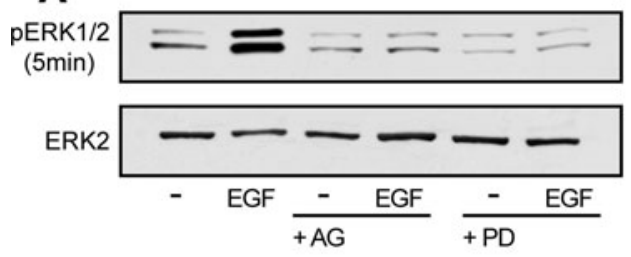

\section{B}

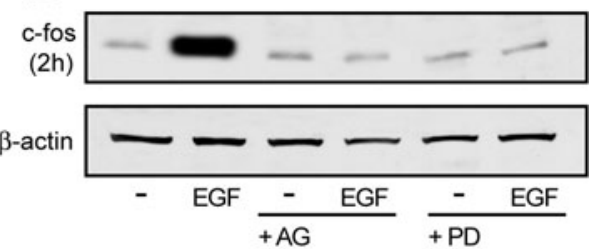

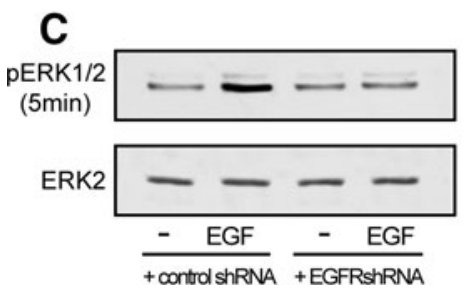
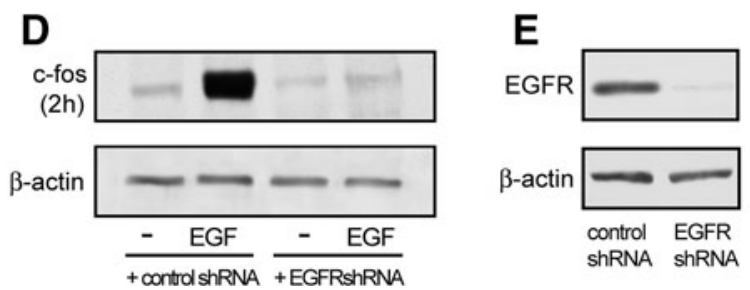

SkBr3

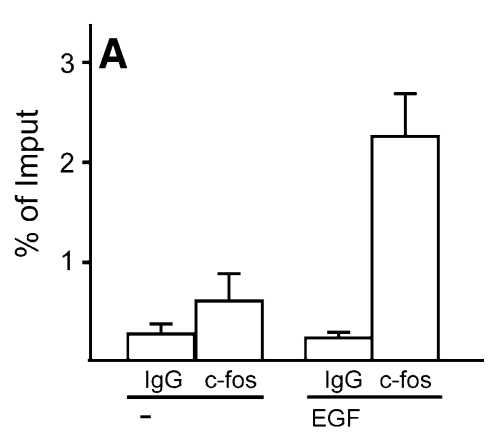

Fig. 6 In $\mathrm{SkBr} 3$ cells, EGF $(50 \mathrm{ng} / \mathrm{ml})$ induces the recruitment of c-fos at the two AP-1 sites corresponding to $-1259 /-1253$ and $-1151 /-1145$ within the SLC37A1 promoter sequence (a), and at the

mitochondria, peroxisomes, and endoplasmic reticulum. Indeed, evaluation of the aminoacid sequence of SLC37A1 protein, performed by PSORT program (http://psort.ims. u-tokyo.ac.jp/form2.html), does not provide information regarding its possible subcellular location(s). However, the absence of peroxisomal target sequences, the presence of endoplasmic reticulum retention sequences, and a mitochondria cleavage site may imply that it may be embedded in the endoplasmic reticulum and/or in the external mitochondrial membrane. Interestingly, in the present study, we have demonstrated that SLC37A1 is localized in the endoplasmic reticulum at least in the cancer cells used as revealed by immunofluorescence evaluation. Since phospholipid biosynthesis, but not glycerol phosphate shuttling, occurs in the endoplasmic reticulum, we can assume that SLC37A1 protein could have a role in the transport of G3P into endoplasmic reticulum to provide phospholipid biosynthesis.

Fatty acid synthase (FAS), the main enzyme involved in de novo fatty acid synthesis, is coordinately regulated at the transcriptional level together with other lipogenic genes to

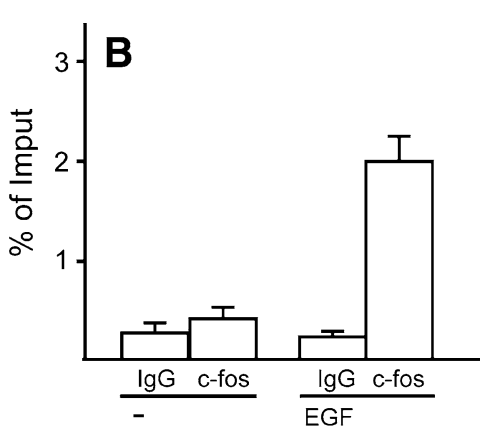

two AP-1 sites, corresponding to $-769 /-763$ and $-747 /-741$ within the SLC37A1 promoter sequence (b). In control samples, non-specific $\mathrm{IgG}$ was used instead of the primary antibody

provide balanced amounts of lipids for membrane biosynthesis [7, 13]. FAS concentration is very low in non-cancerous cells, while it is overexpressed in a wide variety of human cancer cells, supporting the synthesis of phospholipids required for the newly synthesized cellular membrane [31]. Although the ultimate mechanisms responsible for tumor-associated FAS overexpression are not completely understood, growth factor and hormone transduction pathways have been indicated as major contributors. In this regard, FAS expression has been shown to be stimulated by EGF signaling [32], which involves a complex network including activation and/or cross-talk between multiple signal-transduction cascades. For example, MAPK and PI3K pathways are likely candidates for regulating FAS expression in cancer cells through SREBP-1c [33, 34]. Furthermore, Spot14 and p53 family proteins seem to function as transcriptional regulators of genes involved in fatty acid synthesis [21,22]. Although the importance of FAS in de novo phospholipid biosynthesis has been wellrecognized, the contribution of further enzymes/proteins in 


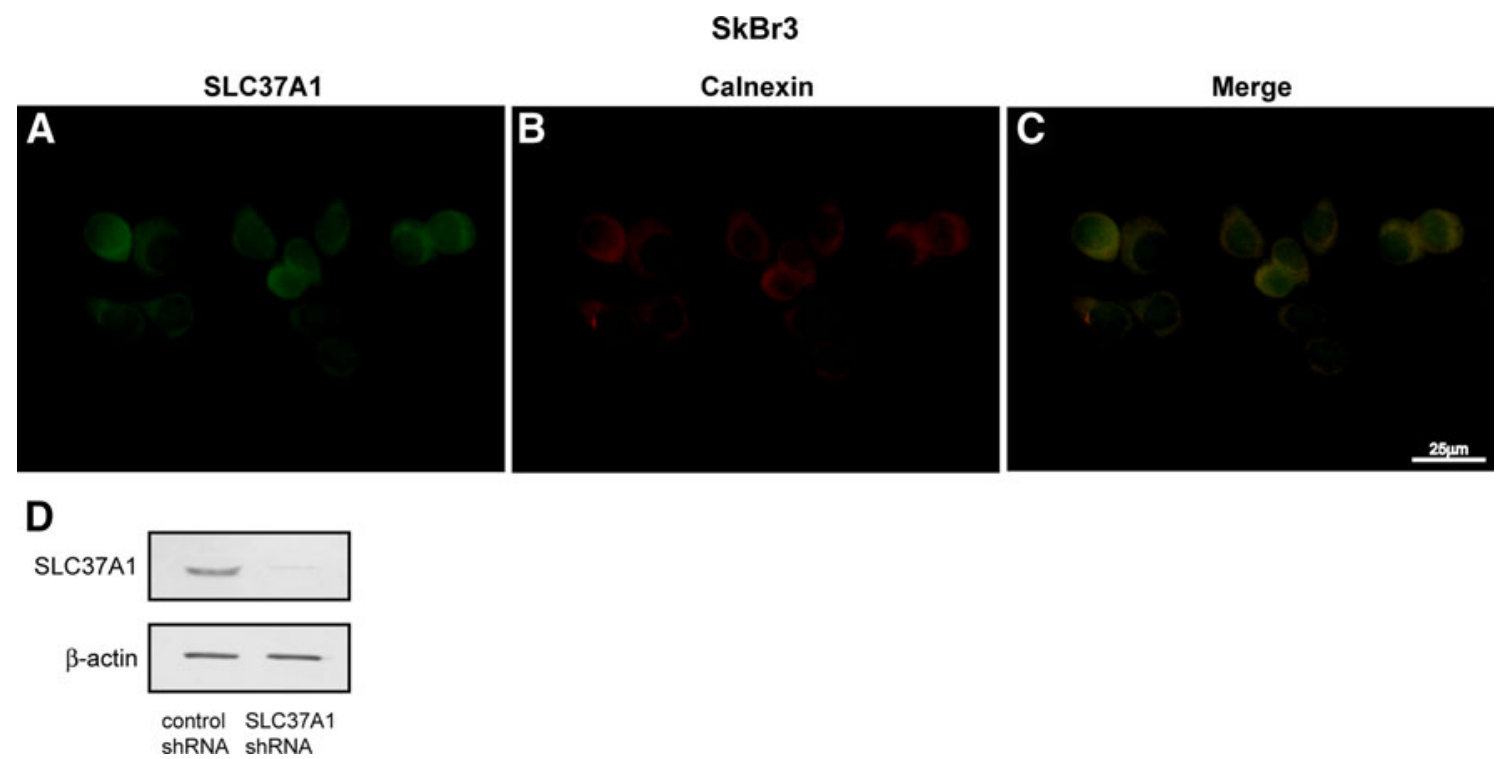

Fig. 7 Subcellular localization of SLC37A1. a Staining of SLC37A1 protein was performed with chicken anti-SLC37A1 monoclonal antibody and rabbit anti-chicken antibody with FITC-conjugated. b Staining of calnexin was performed with rabbit anti-calnexin monoclonal antibody and donkey anti-rabbit antibody with rodamin-conjugated. c Colocalization of the SLC37A1 protein and the

tumor cells remains to be fully evaluated. In this vein, we attempted to characterize the regulation of SLC37A1, a protein potentially involved in phospholipid biosynthesis. Analysing the SLC37A1 promoter sequence, we identified seven AP-1 sites, which may represent the principal targets of growth factors and hormone signaling pathways [35]. Consequently, we have evaluated and demonstrated that SLC37A1 promoter activity is regulated by EGF through the ERK/c-fos transduction pathway in both ER-negative breast $\mathrm{SkBr} 3$ and ER-positive endometrial Ishikawa cancer cells. It is worth to note that among ligands used only EGF was able to up-regulate SLC37A1 expression in SkBr3 cells and, most importantly, in Ishikawa cells characterized by a receptor expression pattern which includes ER $\alpha$, EGFR, and IGF-IR. Hence, the selective response of SLC37A1 to EGF may be consequent to its unique ability to trigger the EGFR/ ERK/c-fos/AP-1 transduction pathway which cannot be activated by either E2 or IGF-1, at least in the cell contexts evaluated. Moreover, the two cancer cell types somehow exhibited a different transcriptional response to EGF using the full length SLC37A1 promoter construct; however, the up-regulation of SLC37A1 expression induced by EGF was comparable in $\mathrm{SkBr} 3$ and Ishikawa cells. Similarly, the SLC37A1 transactivation by EGF in Ishikawa cells is sufficient to trigger SLC37A1 expression levels in a similar extent as observed in $\mathrm{SkBr} 3$ cells.

On the basis of the present findings, the ability of EGF to up-regulate SLC37A1 expression should be included in endoplasmic reticulum is seen as yellow fluorescence in the red and green merged image. Cells were photographed first with a FITC-green filter set, and then with the rodamin-conjugated red filter set. Identical fields are presented. d The specificity of the antibody used was evaluated transfecting $\mathrm{SkBr} 3$ cells with an empty vector or shSLC37A1, as indicated

the well-known stimulatory action exerted by EGF in breast cancer cells. Further studies are needed to provide a better understanding of EGF-mediated SLC37A1 function in breast tumor progression.

Acknowledgments This study was supported by the Associazione Italiana per la Ricerca sul Cancro (AIRC), Ministero dell'Università e Ricerca Scientifica e Tecnologica (MURST) and Regione Calabria.

\section{References}

1. Wakil SJ (1989) Fatty acid synthase, a proficient multifunctional enzyme. Biochemistry 28:4523-4530

2. Coleman RA, Lewin TM, Van Horn CG, Gonzalez-Baro MR (2002) Do long-chain acyl-CoA synthetases regulate fatty acid entry into synthetic versus degradative pathways? J Nutr 132: $2123-2126$

3. Dircks LK, Sul HS (1997) Mammalian mitochondrial glycerol-3phosphate acyltransferase. Biochim Biophys Acta 1348:17-26

4. Gimeno RE, Cao J (2008) Thematic review series: glycerolipids. Mammalian glycerol-3-phosphate acyltransferases: new genes for an old activity. J Lipid Res 49:2079-2088

5. Larson TJ, Schumacher G, Boos W (1982) Identification of the glpT-encoded sn-glycerol-3-phosphate permease of Escherichia coli, an oligomeric integral membrane protein. J Bacteriol 152: 1008-1021

6. Tang W, Yuan J, Chen X, Gu X, Luo K, Li J, Wan B, Wang Y, Yu L (2006) Identification of a novel human lysophosphatidic acid acyltransferase, LPAAT-theta, which activates mTOR pathway. J Biochem Mol Biol 39:626-635

7. Young CD, Anderson SM (2008) Sugar and fat-that's where it's at: metabolic changes in tumors. Breast Cancer Res 10:202 
8. Warburg O, Posener K, Negelein E (1924) Ueber den Stoffwechsel der Tumoren. Reprinted in English in the book On metabolism of tumors by O Warburg (1930), vol 152. Constable, London, pp 319-344

9. Warburg O (1956) On the origin of cancer cells. Science 123 (3191):309-314

10. Menendez JA, Lupu R (2007) Fatty acid synthase and the lipogenic phenotype in cancer pathogenesis. Nat Rev Cancer 7:763-777

11. Kuhajda FP (2000) Fatty-acid synthase and human cancer: new perspectives on its role in tumor biology. Nutrition 16:202-208

12. Kuhajda FP (2006) Fatty acid synthase and cancer: new application of an old pathway. Cancer Res 66:5977-5980

13. Mashima T, Seimiya H, Tsuruo T (2009) De novo fatty-acid synthesis and related pathways as molecular targets for cancer therapy. Br J Cancer 100:1369-1372

14. Cao Y, Dave KB, Doan TP, Prescott SM (2001) Fatty acid CoA ligase 4 is up-regulated in colon adenocarcinoma. Cancer Res 61: 8429-8434

15. Gassler N, Herr I, Schneider A, Penzel R, Langbein L, Schirmacher P, Kopitz J (2005) Impaired expression of acyl-CoA synthetase 5 in sporadic colorectal adenocarcinomas. J Pathol 207: 295-300

16. Diefenbach CS, Soslow RA, Iasonos A, Linkov I, Hedvat C, Bonham L, Singer J, Barakat RR, Aghajanian C, Dupont J (2006) Lysophosphatidic acid acyltransferase-beta (LPAAT-beta) is highly expressed in advanced ovarian cancer and is associated with aggressive histology and poor survival. Cancer 107:1511-1519

17. Bonham L, Leung DW, White T, Hollenback D, Klein P, Tulinsky J, Coon M, de Vries P, Singer JW (2003) Lysophosphatidic acid acyltransferase-beta: a novel target for induction of tumour cell apoptosis. Expert Opin Ther Targets 7:643-661

18. Lupu R, Menendez JA (2006) Targeting fatty acid synthase in breast and endometrial cancer: an alternative to selective estrogen receptor modulators? Endocrinology 147:4056-4066

19. Kumar-Sinha C, Ignatoski KW, Lippman ME, Ethier SP, Chinnaiyan AM (2003) Transcriptome analysis of HER2 reveals a molecular connection to fatty acid synthesis. Cancer Res 63:132-139

20. Menendez JA, Vellon L, Mehmi I, Oza BP, Ropero S, Colomer R, Lupu R (2004) Inhibition of fatty acid synthase (FAS) suppresses HER2/neu (erbB-2) oncogene overexpression in cancer cells. Proc Natl Acad Sci USA 101:10715-10720

21. D'Erchia AM, Tullo A, Lefkimmiatis K, Saccone C, Sbisa E (2006) The fatty acid synthase gene is a conserved p53 family target from worm to human. Cell Cycle 5:750-758

22. Martel PM, Bingham CM, McGraw CJ, Baker CL, Morganelli PM, Meng ML, Armstrong JM, Moncur JT, Kinlaw WB (2006) S14 protein in breast cancer cells: direct evidence of regulation by SREBP-1c, superinduction with progestin, and effects on cell growth. Exp Cell Res 312:278-288
23. Andresen BT, Rizzo MA, Shome K, Romero G (2002) The role of phosphatidic acid in the regulation of the Ras/MEK/Erk signaling cascade. FEBS Lett 531:65-68

24. Fiermonte G, Dolce V, David L, Santorelli FM, Dionisi-Vici C, Palmieri F, Walker JE (2003) The mitochondrial ornithine transporter. Bacterial expression, reconstitution, functional characterization, and tissue distribution of two human isoforms. J Biol Chem 278:32778-32783

25. Morelli C, Garofalo C, Sisci D, del Rincon S, Cascio S, Tu X, Vecchione A, Sauter ER, Miller WH Jr, Surmacz E (2004) Nuclear insulin receptor substrate 1 interacts with estrogen receptor alpha at ERE promoters. Oncogene 23:7517-7526

26. Foster JD, Nordlie RC (2002) The biochemistry and molecular biology of the glucose-6-phosphatase system. Exp Biol Med (Maywood) 227:601-608

27. van Schaftingen E, Gerin I (2002) The glucose-6-phosphatase system. Biochem J 362:513-532

28. Takahashi Y, Miyata M, Zheng P, Imazato T, Horwitz A, Smith JD (2000) Identification of cAMP analogue inducible genes in RAW264 macrophages. Biochim Biophys Acta 1492:385-394

29. Bartoloni L, Antonarakis SE (2004) The human sugar-phosphate/ phosphate exchanger family SLC37. Pflugers Arch 447:780-783

30. Bartoloni L, Wattenhofer M, Kudoh J, Berry A, Shibuya K, Kawasaki K, Wang J, Asakawa S, Talior I, Bonne-Tamir B et al (2000) Cloning and characterization of a putative human glycerol 3-phosphate permease gene (SLC37A1 or G3PP) on 21q22.3: mutation analysis in two candidate phenotypes, DFNB10 and a glycerol kinase deficiency. Genomics 70:190-200

31. Kuhajda FP, Jenner K, Wood FD, Hennigar RA, Jacobs LB, Dick JD, Pasternack GR (1994) Fatty acid synthesis: a potential selective target for antineoplastic therapy. Proc Natl Acad Sci USA 91:6379-6383

32. Swinnen JV, Heemers H, Deboel L, Foufelle F, Heyns W, Verhoeven G (2000) Stimulation of tumor-associated fatty acid synthase expression by growth factor activation of the sterol regulatory element-binding protein pathway. Oncogene 19:5173-5181

33. Van de Sande T, De Schrijver E, Heyns W, Verhoeven G, Swinnen JV (2002) Role of the phosphatidylinositol 3'-kinase/ PTEN/Akt kinase pathway in the overexpression of fatty acid synthase in LNCaP prostate cancer cells. Cancer Res 62:642-646

34. Yang YA, Han WF, Morin PJ, Chrest FJ, Pizer ES (2002) Activation of fatty acid synthesis during neoplastic transformation: role of mitogen-activated protein kinase and phosphatidylinositol 3-kinase. Exp Cell Res 279:80-90

35. Whitmarsh AJ, Davis RJ (1996) Transcription factor AP-1 regulation by mitogen-activated protein kinase signal transduction pathways. J Mol Med 74:589-607 